Name: Peer Review Information for "Isoelectronic IrC3-, PtC3 and AuC3+ Clusters Featuring the Structural and Bonding Resemblance to OC3"

First Round of Reviewer Comments

Reviewer: 1

Comments to the Author

This is an interesting study reporting the similarity of bonding between transition metals IrC3-/PtC3 and OC3 by photoelectron spectroscopy and quantum chemistry calculation. The experimental and theoretical analyses overall are systematic and well done. The analogy of chemical properties between $\mathrm{Pt}$ and $\mathrm{O}$ first proposed by Pyykko is indeed interesting and seems to have been realized in the IrC3-and PtC3 systems. This is an important study and merit publication in JPCL.

Reviewer: 2

\title{
Comments to the Author
}

The authors observed experimentally photoelectron spectra of IrC3- and PtC3- and analyzed the spectra based on DFT calculations. As the photoelectron spectra were reproduced well by the most stable structures, the authors succeeded in determining the geometrical structures. They further calculated structures of PtC3, AuC3+ and OC3 finding that there is similarity in the geometry and bonding natures. The results are interesting and can provide important information for designing new materials.

However, I can question whether these findings are logically connected. In other words, it seems to me that two separate findings are put together, probably because their conclusion may not be lead based on the experiments. I like to suggest that this letter can be published in JPC letter, but the manuscript should be revised such that their findings are logically connected. Experiments, analysis and computation are clear and sound.

Reviewer: 3 


\section{Comments to the Author}

In this combined experimental and theoretical study, the authors investigated the IrC3 - and PtC3 clusters using photoelectron spectroscopy and density functional theory calculations. Striking similarities in the geometries are found for IrC3 - , PtC3, AuC3 + and OC3 complexes. The analysis shows that the IrC3 - , PtC3, AuC3 + and OC3 complexes have analogous chemical bonding properties. The authors therefore concluded that the metal atom can be viewed as oxygen atom in the isoelectronic species (IrC3- , PtC3, AuC3 + ) systems.

The authors presented interesting results in the manuscript. However, this manuscript in its current status lack of relevant information. Therefore, substantial revisions are required. The following points should be addressed.

1. The first two paragraphs on page 3 need to be rephrased to clarify the importance and urgency of this work.

2. In Figure 3, the agreement between the experimental and theoretical results for IrC3- is not obvious.

3. On page 7 , line 52 , the authors wrote "the metal atom can be viewed as oxygen atom in the isoelectronic species (IrC3 - , PtC3, AuC3 + ) systems". This conclusion is not sufficiently convincing. More discussions should be presented.

Author's Response to Peer Review Comments:

Prof. EDITOR

The Journal of Physical Chemistry Letters

Senior Editor
Associate Prof. Hua Xie

Tel.: $\quad+86-411-84379365$

Fax: $\quad+86-411-84379365$

E-Mail: xiehua@dicp.ac.cn 


\section{Prof. EDITOR,}

Thank you very much for your prompt treatment of our manuscript which we submitted for publication in The Journal of Physical Chemistry Letters.

Manuscript ID: jz-2021-03754s

Title: "Isoelectronic $\mathrm{IrC}_{3}^{-}, \mathrm{PtC}_{3}$ and $\mathrm{AuC}_{3}{ }^{+}$Clusters Featuring the Structural and Bonding

Resemblance to $\mathrm{OC}_{3}$ "

Author(s): Liu, Xuegang; Li, Gang; Liu, Zhiling; Yang, Wenshao; Fan, Hongjun; Jiang, Ling; Xie, Hua

We would like to express our sincere thanks to the three reviewers through you for their critical comments and constructive suggestions. We have revised our manuscript according to the valuable suggestions. Attached please find the revised version. Following is the response to the comments from the editor and three reviewers.

Thanks very much for the time and effort that the reviewers and you have given to our manuscript.

Best wishes,

Hua Xie

The response to Editorial Comments for Author (jz-2021-03754s)

\section{General Formatting}

In both the main manuscript file and the Supporting Information, set the Title in title case, with the first letter of each principal word capitalized .

Author reply: We have carefully modified the titles according to the comments. (Pages 1, S1 and S2) 


\section{Table of Content (TOC) graphic}

Please provide a TOC Graphic illustrating the significance of the paper on the same page as the Abstract. The TOC Graphic should be something that is representative of your entire work. Color schemes or illustrations typically make good choices. The TOC Graphic must be original and free from any copyright issues. The TOC Graphic should be approximately 2 in $\mathrm{x} 2$ in and should have adequate resolution and clarity. Confirm that all text is legible at this size. Present the TOC Graphic on the same page as the Abstract with the heading "TOC Graphic” . A caption describing the TOC

Graphic is not needed.

Author reply: The TOC graphic has been placed after the Abstract. (Page 2)

\section{References}

In both the main file and the supporting information, fix the style of all references to use JPCL formatting (check all references carefully). ***JPC Letters reference formatting requires that journal references should contain: () around numbers, author names, article title (titles entirely in title case or entirely in lower case), abbreviated journal title (italicized), year (bolded), volume (italicized), and pages (first-last). Book references should contain author names, book title (in the same pattern), publisher, city, and year.

Author reply: The references have been carefully revised according to the JPC Letters formatting. (Pages 9-10)

\section{The response to the comments from Referee \#1:}

Recommendation: This paper represents a significant new contribution and should be published as is. Comments:

This is an interesting study reporting the similarity of bonding between transition metals $\mathrm{IrC}_{3}^{-} /$ $\mathrm{PtC}_{3}$ and $\mathrm{OC}_{3}$ by photoelectron spectroscopy and quantum chemistry calculation. The experimental and theoretical analyses overall are systematic and well done. The analogy of chemical properties between Pt and O first proposed by Pyykko is indeed interesting and seems to have been realized in the $\mathrm{IrC}_{3}{ }^{-}$and $\mathrm{PtC}_{3}$ systems. This is an important study and merit publication in JPCL. 
Author reply: Thanks for the reviewer's comments.

The response to the comments from Referee $\# 2$ :

Recommendation: This paper is probably publishable, but major revision is needed; I do not need to see future revisions.

Comments:

The authors observed experimentally photoelectron spectra of $\mathrm{IrC}_{3}{ }^{-}$and $\mathrm{PtC}_{3}{ }^{-}$and analyzed the spectra based on DFT calculations. As the photoelectron spectra were reproduced well by the most stable structures, the authors succeeded in determining the geometrical structures. They further calculated structures of $\mathrm{PtC}_{3}, \mathrm{AuC}_{3}{ }^{+}$and $\mathrm{OC}_{3}$ finding that there is similarity in the geometry and bonding natures. The results are interesting and can provide important information for designing new materials.

However, I can question whether these findings are logically connected. In other words, it seems to me that two separate findings are put together, probably because their conclusion may not be lead based on the experiments. I like to suggest that this letter can be published in JPC letter, but the manuscript should be revised such that their findings are logically connected. Experiments, analysis and computation are clear and sound.

Author reply: The analogy of chemical properties between each of $\mathrm{Pt}, \mathrm{Au}^{+}$and $\mathrm{O}$ atom first proposed by Pyykkö is indeed interesting. Since $\mathrm{Ir}^{-}, \mathrm{Ptand}^{+} \mathrm{u}^{+}$have the same number of electrons, we assume that $\mathrm{Ir}^{-}$may have the similar chemical properties to the $\mathrm{Pt}$ and $\mathrm{Au}^{+}$. Previous studies have indicated that $\mathrm{PtC}_{3}$ and $\mathrm{AuC}_{3}{ }^{+}$are linear structures with $\mathrm{MCCC}$ characteristic. To verify our assumption, photoelectron spectra and quantum calculation are performed to study the $\mathrm{MC}_{3}^{-}$ $\left(\mathrm{M}=\mathrm{Ir}\right.$ and $\mathrm{Pt}$ ) clusters. We find that $\mathrm{IrC}_{3}{ }^{-}$and $\mathrm{PtC}_{3}$ have the similar structures as $\mathrm{AuC}_{3}{ }^{+}$and $\mathrm{OC}_{3}$. Our further calculations have showed that $\mathrm{IrC}_{3}^{-}, \mathrm{PtC}_{3}$ and $\mathrm{AuC}_{3}{ }^{+}$have similar bonding behavior as $\mathrm{OC}_{3}$.

(Page 6)

The response to the comments from Referee \#3: 
Recommendation: This paper may be publishable, but major revision is needed; I would like to be invited to review any future revision Comments:

In this combined experimental and theoretical study, the authors investigated the $\mathrm{IrC}_{3}{ }^{-}$and $\mathrm{PtC}_{3}{ }^{-}$ clusters using photoelectron spectroscopy and density functional theory calculations. Striking similarities in the geometries are found for $\mathrm{IrC}_{3}^{-}, \mathrm{PtC}_{3}, \mathrm{AuC}_{3}{ }^{+}$and $\mathrm{OC}_{3}$ complexes. The analysis shows that the $\mathrm{IrC}_{3}^{-}, \mathrm{PtC}_{3}, \mathrm{AuC}_{3}{ }^{+}$and $\mathrm{OC}_{3}$ complexes have analogous chemical bonding properties. The authors therefore concluded that the metal atom can be viewed as oxygen atom in the

isoelectronic species $\left(\mathrm{IrC}_{3}^{-}, \mathrm{PtC}_{3}, \mathrm{AuC}_{3}{ }^{+}\right)$systems.

The authors presented interesting results in the manuscript. However, this manuscript in its current status lack of relevant information. Therefore, substantial revisions are required. The following points should be addressed.

1) The first two paragraphs on page 3 need to be rephrased to clarify the importance and urgency of this work.

Author reply: Thanks for the reviewer's comments. We have carefully modified the manuscript to highlight the important of this study. Gas cluster has the advantages of isolation, controllability and repeatability. Photoelectron spectroscopy combined with theoretical calculation can not only obtain the formation and distribution of the clusters, but also the structure and reactivity of the clusters. Furthermore, the active site and microscopic mechanism of the reaction can be revealed at the level of chemical bond and electronic structures. (Page 3)

2) In Figure 3, the agreement between the experimental and theoretical results for $\mathrm{IrC}_{3}{ }^{-}$is not obvious.

Author reply: Thanks for the reviewer's comments. For $\mathrm{IrC}_{3}{ }^{-}$, our extensive geometrical optimizations lead to the linear ground state (3A) with an Ir atom terminally bonded to the linear $\mathrm{C}_{3}$ chain. Alternative optimized anionic structures are predicted to be at least $\sim 1.0 \mathrm{eV}$ higher in energy, hinting at the overwhelming stability of the linear structure 3A. Usually, the 
thermodynamically unfavorable isomers can not easily survive under our cluster source conditions. Moreover, the isomer 3B and 3C in higher energy could be ruled out because their VDE values disagree with the experimental values. Besides, the PES of the top three lowest-lying isomers are simulated and compared with the experimental one (Fig. 3). The simulated spectra are constructed by fitting the distribution of calculated vertical detachment energy values with unitarea Gaussian functions of $0.10 \mathrm{eV}$ width, without the consideration of the vibrational excitation. Nevertheless, the simulated PES profile of $3 \mathrm{~A}$ fits the best with the observed PES spectra features.

3) On page 7 , line 52 , the authors wrote "the metal atom can be viewed as oxygen atom in the isoelectronic species $\left(\mathrm{IrC}_{3}^{-}, \mathrm{PtC}_{3}, \mathrm{AuC}_{3}^{+}\right)$systems". This conclusion is not sufficiently convincing. More discussions should be presented.

Author reply: Thanks for the reviewer's comments. The concept of chemical properties between $\mathrm{Pt}$ and $\mathrm{O}$ analogy was first proposed by Pyykkö et al. The $\mathrm{Pt}_{3} \mathrm{C}^{+}$cluster ion has been observed by infrared multiple photon dissociation (IRMPD) spectroscopy, whose geometry was similar to carbonate ion consistent with the empirical model predictions. (J. Phys. Chem. Lett. 2013, 4, 892-896) In addition, the similarity between $\mathrm{PtC}_{3}$ and $\mathrm{OC}_{3}$ has been also proved by rotational spectroscopy. (Angew. Chem. Int. Ed. 2016, 55, 3768-3771). Meanwhile, $\mathrm{AuC}_{3}{ }^{+}$has been also proved to be a straight chain structure by the previous experiments. (J. Phys. Chem. A 2008, 112, 12355-12366). Previous theoretical calculations have revealed analogies between the behavior of each of $\mathrm{Au}^{+}$, Pt and $\mathrm{O}$ atom. ( Chem. Phys. Lett. 2003, 381, 45-52). Due to $\mathrm{IrC}_{3}{ }^{-}, \mathrm{PtC}_{3}$ and $\mathrm{AuC}_{3}{ }^{+}$ clusters have the same number of electrons, if the $\mathrm{IrC}_{3}{ }^{-}, \mathrm{PtC}_{3}$ and $\mathrm{AuC}_{3}{ }^{+}$have the similar structures, they could be isoelectronic. The current theoretical calculations have indicated that the most stable structures of the $\mathrm{IrC}_{3}{ }^{-}, \mathrm{PtC}_{3}$ and $\mathrm{AuC}_{3}{ }^{+}$are straight chain structures with an MCCC characteristic. Therefore, the $\mathrm{IrC}_{3}{ }^{-}, \mathrm{PtC}_{3}$ and $\mathrm{AuC}_{3}{ }^{+}$clusters could be isoelectronic. Since Pt and $\mathrm{Au}^{+}$can be viewed as analogy to $\mathrm{O}$ atom, similarly, $\mathrm{Ir}^{-}$may be analogic to $\mathrm{O}$ atom. Further calculations have showed that they have similar bonding behavior. Additionally, combining with MOs, AdNDP and NBO analysis, the analogic bond features are also found in $\mathrm{OC}_{3}$. The analogy of chemical properties is indeed interesting and seems to have been realized in the $\mathrm{IrC}_{3}{ }^{-}, \mathrm{PtC}_{3}$ and 
$\mathrm{AuC}_{3}{ }^{+}$systems. This will be a new concept about isoelectronic species $\left(\mathrm{Ir}^{-}, \mathrm{Pt}\right.$, and $\left.\mathrm{Au}^{+}\right)$-oxygen analogy. (Pages 7-8) 